\title{
Influence of nanocrystal growth kinetics on interface roughness in nickel-aluminum multilayers
}

\author{
D. Aurongzeb and M. Holtz ${ }^{\text {a) }}$ \\ Department of Physics and NanoTech Center, Texas Tech University, Lubbock, Texas 79409 \\ M. Daugherty and J. M. Berg \\ Department of Mechanical Engineering and NanoTech Center, Texas Tech University, Lubbock, Texas 79409
}

A. Chandolu, J. Yun, and H. Temkin

Department of Electrical Engineering and NanoTech Center, Texas Tech University, Lubbock, Texas 79409

(Received 25 August 2003; accepted 3 November 2003)

\begin{abstract}
We study the layer morphology of Ni/Al multilayer structures, with $50 \mathrm{~nm}$ period, as deposited and following $10 \mathrm{~min}$ anneals up through the melting temperature of Al. X-ray reflectivity measurement of the as-deposited film shows interference fringes, characteristic of a well-defined multilayer stack, with $\sim 1 \mathrm{~nm}$ interface roughness. Over a narrow anneal range of $360-500{ }^{\circ} \mathrm{C}$ these fringes diminish in amplitude and disappear, indicating elevated interface roughening. However, fringes are observed for anneal temperatures both below and above this range, indicating the presence of well-defined layers with smooth interfaces. A model, in which nanocrystal domains of intermetallic nickel aluminides form at the interfaces, is developed to quantify the annealing induced interface roughness. This model agrees well with the experimental results. (C) 2003 American Institute of Physics. [DOI: 10.1063/1.1637155]
\end{abstract}

Intermetallics, such as those produced from pure aluminum and nickel precursors, are important for application in corrosion and oxidation resistant coatings. ${ }^{1-5}$ One interesting method by which to produce precursor materials and study formation properties is to deposit multiple layers of pure $\mathrm{Ni}$ and $\mathrm{Al}$ with short bilayer periods. Annealing Ni/Al multilayers from room temperature to above the $\mathrm{Al}$ melting point $\left(660^{\circ} \mathrm{C}\right)$, but well below that of $\mathrm{Ni}\left(1453^{\circ} \mathrm{C}\right)$, produces interesting results due to the variety of possible stable alloy phases and energetic release due to solid-state reactions. Because of the high surface to volume interaction ratios in nanometer-scale structures, the study of surface and interface properties is currently important from both scientific and applied viewpoints. ${ }^{6,7}$

The majority of published studies have reported x-ray diffraction (XRD) to address compositional changes caused by annealing in various temperature ranges. ${ }^{8-13}$ Additionally, differential scanning calorimetry (DSC) has been used for examining the energetic release from the formation of stable phases upon heating. ${ }^{10-12} \mathrm{Ma}$ et al. applied the model of Coffey et al. ${ }^{14}$ to their Ni/Al multilayer studies by considering domain formation where grain boundaries meet the $\mathrm{Ni}-\mathrm{Al}$ interfaces. For the $\mathrm{Nb} / \mathrm{Al}$ and $\mathrm{Ni} / a-\mathrm{Si}^{14}$ and the $\mathrm{Ni} / \mathrm{Al}$ experiments ${ }^{10}$ this model accurately describes the DSC results.

We have found few papers ${ }^{8,9}$ that focus on the interface properties of $\mathrm{Ni} / \mathrm{Al}$ multilayers, and none that addresses the effect of nanocrystal domain formation on the interface roughness of multilayer stacks. We report the effects of annealing on interface properties using $\mathrm{Ni} / \mathrm{Al}$ as the prototype system.

The Ni/Al multilayer samples were prepared by electron

${ }^{a)}$ Electronic mail: mark.holtz@ttu.edu beam evaporation on glass and silicon substrate wafers. The evaporator base pressure was $\leqslant 10^{-7}$ Torr. Multilayers were deposited by successively alternating $\mathrm{Ni}$ and $\mathrm{Al}$ sources, with the deposition rate for each $\sim 1 \AA$ s. A total of 20 bilayers were deposited with individual layer thickness $L=25 \mathrm{~nm}$ for a $50 \mathrm{~nm}$ period and total thickness $\sim 1 \mu \mathrm{m}$. The $50 \mathrm{~nm}$ period was chosen because it is reported to give the maximum energetic release upon self-propagating reaction. ${ }^{1}$ A scanning electron microscope (SEM) cross section of our as-deposited stack is shown in Fig. 1. Clearly seen is a well-defined multilayer structure that exhibits a $52 \pm 2 \mathrm{~nm}$ period, in close agreement with the $50 \mathrm{~nm}$ target.

After deposition the samples were annealed in a conventional furnace at temperatures ranging from 260 to $660^{\circ} \mathrm{C}$ in $100^{\circ} \mathrm{C}$ intervals. This range includes the melting point of aluminum at $660^{\circ} \mathrm{C}$ and overlaps ranges of interest in published work. ${ }^{12,15,16}$ Samples were transferred into a preheated oven to obtain a rapid temperature ramp. This procedure is used to avoid the formation of stable phases below the desired anneal temperature upon slowly heating. The annealing atmosphere was nitrogen gas. The $10 \mathrm{~min}$ anneal allows us to capture the solid-state reaction in its early stages. ${ }^{10}$

Figure 2 shows $\mathrm{X}$-ray reflectivity (XRR) measurements of samples from the same deposition but following $10 \mathrm{~min}$ anneals across the range of temperatures studied. We analyzed the XRR measurements based on the position and separation of critical angles. In Fig. 2 we observe two critical angles at $2 \theta_{c}$ values of $0.46^{\circ}$ and $0.82^{\circ}$ for the as-deposited film. The smaller critical angle is from the aluminum component of the multilayer and the larger value stems from the denser nickel. ${ }^{17}$ With an increase in anneal temperature, these critical angles are seen to merge as the two distinct materials form intermetallic compounds and, eventually, become a single layer. 


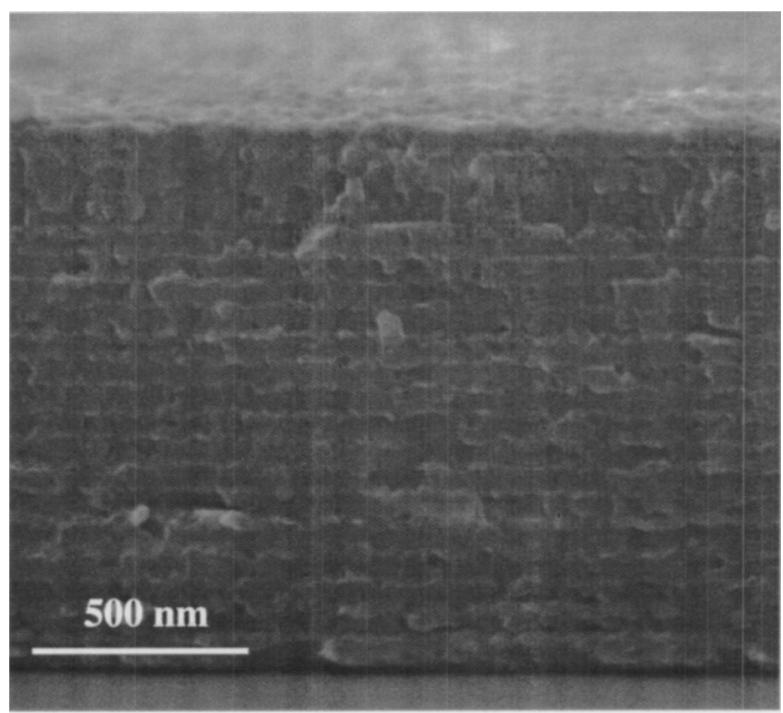

FIG. 1. SEM cross section of as-deposited Ni/Al multilayer foil.

We focus in this letter on the interference fringes above $2 \theta_{c}$, which are due to the multilayer structure. As seen from the data in Fig. 2, XRR fringes are present at each temperatures below the $\mathrm{Al}$ melting point of $660^{\circ} \mathrm{C}$, but with noticably diminished amplitude at $360{ }^{\circ} \mathrm{C}$ and completely absent at $460{ }^{\circ} \mathrm{C}$. This reproducible result is very interesting, because it is consistent with a narrow temperature range over which interface roughness is very high, while sharp interfaces are present both below and above this range. We use a recent Fourier transform approach based on fringe spacing to obtain multilayer periodicity. ${ }^{18}$ In all cases we obtain a bilayer period ranging from 48.4 to $54.4 \mathrm{~nm}$.

Interface roughness properties were determined from the XRR fringe amplitudes. ${ }^{19}$ The as-deposited and $260{ }^{\circ} \mathrm{C}$ anneal results give interface roughness values of $1.1 \pm 0.4 \mathrm{~nm}$. These values are very close to the roughness of both our glass and silicon substrates 1 to $2 \mathrm{~nm}$ according to atomic force microscopy (AFM). We conclude that the interface roughness of the as-deposited stack resembles that of the starting substrate and does not change substantially during growth or due to low-temperature annealing. Fringe analysis gives interface roughness values of $2.0 \pm 0.6$ and 1.4 $\pm 0.4 \mathrm{~nm}$ for 500 and $560{ }^{\circ} \mathrm{C}$ anneals, respectively. The modest increase in interface roughness from that found for the as-deposited layers indicates that the layers are smooth even following these high temperature anneals. Experimental interface roughness values are shown in Fig. 3.

The absence of XRR fringes in a narrow temperature range around $460{ }^{\circ} \mathrm{C}$ can be attributed to the formation of nanocrystal domains. XRD measurements of our samples identifies the domains as intermetallic compositions, $\mathrm{Al}_{3} \mathrm{Ni}_{2}$ and $\mathrm{AlNi}$ phases, following the lower temperature anneals, and $\mathrm{Al}_{3} \mathrm{Ni}_{2}$ and $\mathrm{AlNi}_{3}$ after higher temperature anneals. ${ }^{18}$ Domains are plausibly assumed to grow at the Ni/Al interfaces as illustrated in the inset of Fig. 3. We now develop a model for interface roughness based on previous DSC studies of Ni/Al multilayers. ${ }^{10}$ This model interprets exothermic solid-solid reactions via the formation of nanocrystal domains of intermetallics at interfaces. The domains are taken as having a cylindrical shape, as illustrated in the Fig. 3 Downloaded 07 Jan 2004 to 129.118.119.85. Redistribution subject

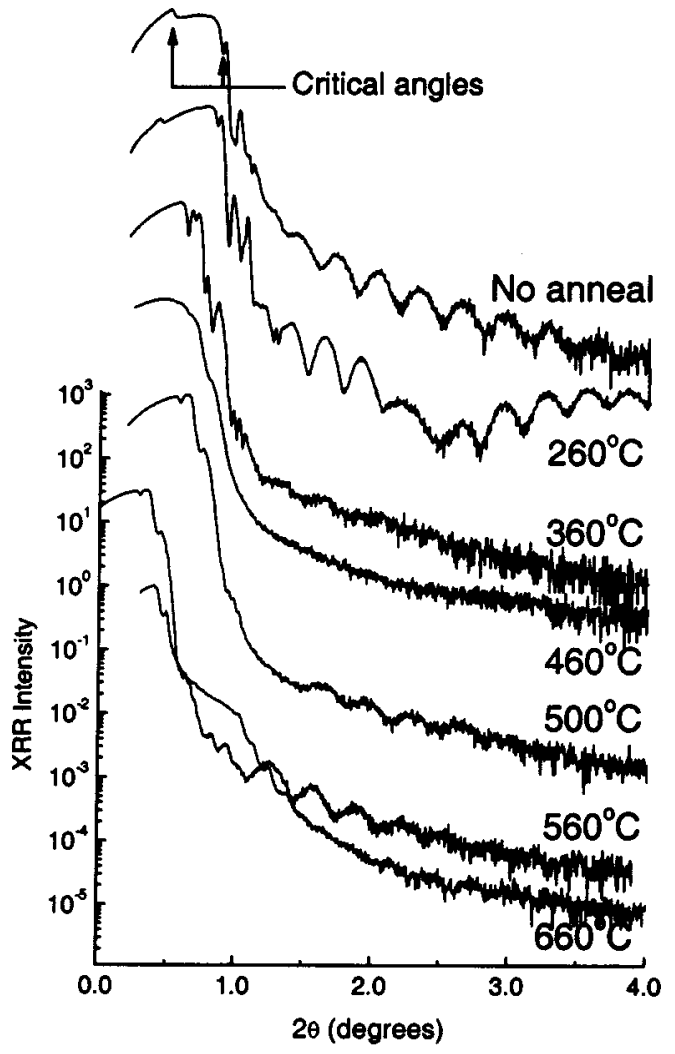

FIG. 2. XRR summary of Ni/Al stacks annealed at various temperatures. The data are offset for illustration purposes.

inset. In the growth plane (perpendicular to the layer normal), the cylinder radius $R$ grows with velocity,

$$
\frac{d R}{d t}=K_{\perp} \exp \left(-E_{\perp} / k T\right),
$$

where $K_{\perp}$ and $E_{\perp}$ are the interface growth prefactor and activation energy, respectively. Integration gives $R \propto t$ so the area covered by each cylinder grows like $t^{2}$. Radial growth of the domains produces an areal coverage fraction $f_{A}$ given by

$$
f_{A}=1-\exp \left(-n \pi\langle R\rangle^{2}\right),
$$

where $n$ is the areal density of domain nucleation sites and $\langle R\rangle$ is the average domain radius. In analyzing the DSC studies, nucleation occurs where grain boundaries intersect interfaces so $n$ is taken to be the areal grain boundary density. In our case, the grains are of the order of the individual layer thickness. ${ }^{10}$ The growth velocity along the layer normal $(z$ direction) relies on atoms from the pure layers diffusing through the existing domain, of thickness $z$, to reach the surface of the nanocrystal. In the case of $\mathrm{Ni} / \mathrm{Al}, \mathrm{Ni}$ will be the faster diffuser, since the Al melting point is much lower than that of $\mathrm{Ni}^{20}$ The domain height grows according to

$$
\frac{d z}{d t}=\frac{1}{z} K_{\|} \exp \left(-E_{\|} / k T\right)
$$

where $K_{\|}$and $E_{\|}$are the diffusion-limited growth prefactor and activation energy, respectively. Integration yields $z \propto \sqrt{t}$, as expected for diffusion-limited growth. Using the parameters obtained by Ma et al., ${ }^{10}$ each of the above can be computed for Ni/Al. Depending on the parameters of this model, to AIP license or copyright, see http://ojps.aip.org/aplo/aplcr.jsp 


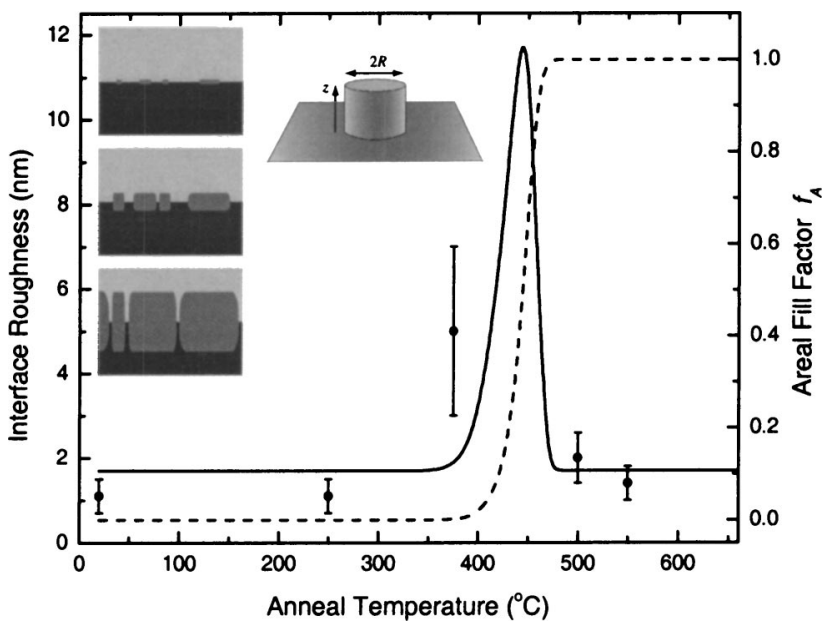

FIG. 3. Anneal temperature dependence of calculated induced interface roughness (solid curve) and areal fill factor (dashed curve). The data are taken from XRD fringe analysis except for that at $360{ }^{\circ} \mathrm{C}$, which was estimated from the SEM cross section. The inset series on the left depicts growth of the nanocrystals. The inset on the right shows the cylindrical geometry used to model nanocrystal domain formation.

perpendicular growth may exceed in-plane growth and produce features with high aspect ratio. Conditions for which the in-plane domain growth is significantly faster than domain height growth will cause lateral domain coalescence, thus preserving the interface smoothness. The $t^{2}$ dependence in $\langle R\rangle$ will cause $f_{A}$ to rapidly transition from zero to unity, thereby exhibiting either sparse or full areal coverage below and above the transition region, respectively.

We apply this model to address the induced roughness of an arbitrarily chosen interface using the standard root-meansquare (rms) roughness $\sigma$ according to

$$
\sigma^{2}-\sigma_{0}^{2}=\frac{\iint_{A}(z(x, y)-\bar{z})^{2} d x d y}{A},
$$

where $x$ and $y$ are coordinates in the interface plane and $A$ is an area sufficiently large to include many domains and provide a statistically meaningful measure of roughness. $\bar{z}$ is the average height of the domains taken from the interface. For $\sigma_{0}$ we use the rms roughness of the substrate, $\sim 1.7$ $\pm 0.5 \mathrm{~nm}$. Using $\langle z\rangle$ to represent the average height of a domain, calculated using Eq. (3), we arrive at

$$
\sigma_{\text {norm }}^{2}=\frac{\sigma^{2}-\sigma_{0}^{2}}{L^{2}}=f_{A}\left(1-f_{A}\right)\left(\frac{\langle z\rangle}{L}\right)^{2} \text {. }
$$

We have divided by $L^{2}$ to obtain a normalized quantity.

Figure 3 shows $f_{A}$ and $\sigma$ calculated from room temperature through the $\mathrm{Al}$ melting point. We use the Ni/Al parameters from Ref. 10: $K_{\|}=0.66 \mathrm{~cm}^{2} / \mathrm{s}, E_{\|}=1.49 \mathrm{eV}$, and $n K_{\perp}^{2}$ $=2.5 \times 10^{24} \mathrm{~s}^{-2}$. $E_{\perp}$ between 2.0 and $2.2 \mathrm{eV}$ best describes our results, but it is slightly higher than the value of 1.44 $\pm 0.1 \mathrm{eV}$ reported in Ref. 10. $f_{A}$ shows the expected sigmoid-like dependence, so the quantity $f_{A}\left(1-f_{A}\right)$ in Eq. (5) exhibits a peak profile with temperature and width sensitive to $E_{\perp}$. Our calculation is for $t=10 \mathrm{~min}$; shorter anneal times make the $f_{A}$ transition more gradual and shift the temperature at which it reaches unity toward higher values. The ratio $\langle z\rangle / L$ in Eq. (5) increases from zero to one at a temperature related to the domain-height activation energy $E_{\|}$. The interface roughness $\sigma$ in Fig. 3 shows a sharp feature at $\sim 460{ }^{\circ} \mathrm{C}$. The induced roughness is zero from room temperature to $\sim 350^{\circ} \mathrm{C}$ and from $\sim 480^{\circ} \mathrm{C}$ through the $\mathrm{Al}$ melting point. In other words, the layer's smoothness is not influenced by annealing at these temperatures. This agrees with our XRR results. In the narrow temperature range between 350 and $480^{\circ} \mathrm{C}$ the model predicts significant induced interface roughness. The peak induced roughness value of $\sim L / 2$ at $\sim 450{ }^{\circ} \mathrm{C}$ is consistent with the total absence of XRR interference fringes. Our XRR data in Fig. 2 show that there is diminished fringe amplitude following the $360^{\circ} \mathrm{C}$ anneal and no fringes for the $460{ }^{\circ} \mathrm{C}$ case. The calculated temperature dependence of $\sigma$, based on this simple model, thus describes well the observed temperature dependence of interface smoothness in $\mathrm{Ni} / \mathrm{Al}$ multilayers.

In summary, we observed an interesting dependence of the interface smoothness of Ni/Al multilayer stacks following annealing at different temperatures. A very narrow temperature range is found in XRR measurements over which no interference fringes are observed, implying rough interfaces. We developed a roughness model, based on the formation of nanocrystal domains. ${ }^{10}$ This model provides a clear explanation for the effect observed.

The authors thank M. Pantoya, N. Güven, and L. Grave de Peralta for helpful discussions. This work was supported in part by a grant from the National Science Foundation (Grant No. CTS 0210141).

${ }^{1}$ A. J. Gavens, D. van Heerden, A. B. Mann, M. E. Reiss, and T. P. Weihs, J. Appl. Phys. 87, 1255 (2000).

${ }^{2}$ E. Besnoin, S. Cerutti, O. M. Knio, and T. P. Weihs, J. Appl. Phys. 92, 5474 (2002).

${ }^{3}$ B. K. Paul, H. Hasan, T. Dewey, D. Alman, and R. D. Wilson, Proc. IMEC 2002, ASME p. 1.

${ }^{4}$ G. M. Poletaev and M. D. Starostenkov, Tech. Phys. Lett. 29, 454 (2003).

${ }^{5}$ Z. Z. Mutasim, Turbomachinery Technology Seminar, Solar Turbines Incorporated, 1998, Vol. 117, p. 1.

${ }^{6}$ J. K. Bording, B. Q. Li, Y. F. Shi, and J. M. Zou, Phys. Rev. Lett. 90, 226104 (2003).

${ }^{7}$ F. B. de Mongeot, W. Zhu, A. Molle, R. Buzio, C. Borgano, U. Valbusa, E. G. Wang, and Z. Zhang, Phys. Rev. Lett. 91, 016102 (2003).

${ }^{8}$ E. Fonda, F. Petroff, and A. Traverse, J. Appl. Phys. 93, 5937 (2003).

${ }^{9}$ U. Rothhaar, H. Oechsner, M. Scheib, and R. Müller, Phys. Rev. B 61, 974 (2000).

${ }^{10}$ E. Ma, C. V. Thompson, and L. A. Clevenger, J. Appl. Phys. 69, 2211 (1991).

${ }^{11}$ A. S. Edelstein, R. K. Everett, G. Y. Richardson, S. B. Qadri, E. I. Altman, J. C. Foley, and J. H. Perepezko, J. Appl. Phys. 76, 7850 (1994).

${ }^{12}$ C. Michaelson, G. Lucadamo, and K. Barmak, J. Appl. Phys. 80, 6689 (1996).

${ }^{13}$ E. G. Colgan, M. Nastasi, and J. W. Mayer, J. Appl. Phys. 58, 4125 (1985).

${ }^{14}$ K. R. Coffey, K. Barmak, and D. A. Rudman, J. Appl. Phys. 72, 1341 (1992).

${ }^{15}$ J. C. Liu, J. W. Mayer, and J. C. Barbour, J. Appl. Phys. 64, 656 (1988).

${ }^{16}$ F. Chevrier, A. Delobbe, A. Traverse, and D. Zanghi, J. Appl. Phys. 90, 2718 (2001).

${ }^{17}$ M. A. Marcus, Appl. Phys. Lett. 72, 659 (1998).

${ }^{18}$ L. Grave de Peralta and H. Temkin, Appl. Phys. Lett. 93, 1974 (2003).

${ }^{19}$ H. J. Voorma, E. Louis, N. B. Koster, F. Bijkerk, and E. Spiller, J. Appl. Phys. 81, 6112 (1997).

${ }^{20}$ R. W. Bené, Appl. Phys. Lett. 41, 529 (1982). 